\title{
Guest Editorial: Clarifications on the use of the new classification of periodontitis
}

\begin{abstract}
This editorial provides clarifications on the application of the Stage and Grade classification of periodontitis. In particular it describes: (1) how to apply the extent criterion to the defined Stage of the disease; and (2) how to calculate tooth loss because of periodontitis in Stage III and IV cases presenting with evidently hopeless (irrational to treat) teeth with a clinical definition of such teeth.
\end{abstract}

One component of the new periodontitis classification (Papapanou et al., 2018) has raised some questions relative to the specific criteria for "extent" in the staging process. The question is whether extent should describe those teeth that harbor the most severe sites that define the patient's stage or those teeth with any degree of attachment loss.

We support the criteria defined in the consensus publication (Papapanou et al., 2018; Tonetti, Greenwell, \& Kornman, 2018), where it is stated that the extent should be described after the determination of the staging (For each stage, describe extent as localized $[<30 \%$ of teeth involved], generalized, or molar/incisor pattern.). Following these criteria, "extent" refers to the stage that captures the overall severity and complexity of the case. Therefore, assessment of extent after the stage has been determined describes the percentage of teeth at the stage-defining severity level and conveys meaningful information to the clinician, as it depicts the percentage of teeth that are severely affected and may likely require treatment of higher complexity.

Another matter of concern is whether to consider as lost teeth because of periodontitis, those existing teeth with an evident hopeless prognosis. We support the inclusion of evidently hopeless teeth when calculating the number of teeth lost because of periodontitis for distinguishing between Stages III and IV periodontitis. It is, however, important to define appropriately what is a hopeless tooth (also termed irrational to treat). Evident hopeless teeth are those in which the attachment loss approximates the apex of the root

This article has been co-published with permission in Journal of Periodontology and Journal of Clinical Periodontology. The articles are identical except for minor stylistic and spelling differences in keeping with each journal's style. Either citation can be used when citing this article. circumferentially, in combination with a high degree of tooth hypermobility (degree III).

\section{KEYWORDS}

classification, extent, grade, periodontitis, stage, tooth loss

Mariano Sanz ${ }^{1}$

Panos N. Papapanou ${ }^{2}$

Maurizio S. Tonetti ${ }^{3,4}$

Henry Greenwell ${ }^{5}$

Kenneth Kornman ${ }^{6}$ iD

${ }^{1}$ ETEP Research Group, Faculty of Dentistry, Complutense University of Madrid, Madrid, Spain

${ }^{2}$ Division of Periodontics, Section of Oral, Diagnostic and Rehabilitation Sciences, College of Dental Sciences, Columbia

University, New York, New York

${ }^{3}$ European Research Group on Periodontology, Genova, Italy

${ }^{4}$ Division of Periodontology and Implant Dentistry, Faculty of Dentistry, The University of Hong Kong, Hong Kong, Hong Kong,

SAR, China

${ }^{5}$ Graduate Periodontics, School of Dentistry, University of Louisville, Louisville, Kentucky

${ }^{6}$ Department of Periodontics and Oral Medicine, University of Michigan School of Dentistry, Ann Arbor, Michigan

Correspondence

Professor Mariano Sanz, ETEP Research Group, Faculty of Odontology, Complutense University of Madrid, Plaza Ramon y Cajal s/n, 28040 Madrid -- Spain. Email: marsan@ucm.es

\section{ORCID}

Mariano Sanz (iD https://orcid.org/0000-0002-6293-5755

Panos N. Papapanou (iD https://orcid.org/0000-0002-6538-3618

Maurizio S. Tonetti iD https://orcid.org/0000-0002-2743-0137

Kenneth Kornman iD https://orcid.org/0000-0003-2252-7324 


\section{REFERENCES}

Papapanou, P. N., Sanz, M., Buduneli, N., Dietrich, T., Feres, M., Fine, D. H., ... Tonetti, M. S. (2018). Periodontitis: Consensus report of workgroup 2 of the 2017 world workshop on the classification of periodontal and peri-implant diseases and conditions. Journal of

Periodontology, 89(Suppl 1), S173-S182. https://doi.org/10.1002/ JPER.17-0721

Tonetti, M. S., Greenwell, H., \& Kornman, K. S. (2018). Staging and grading of periodontitis: Framework and proposal of a new classification and case definition. Journal of Periodontology, 89(Suppl 1), S159-S172. https://doi.org/10.1002/JPER.18-0006 